\title{
CRAVO-DE-DEFUNTO COMO PLANTA ATRATIVA PARA TRIPES EM CULTIVO PROTEGIDO DE MELÃO ORGÂNICO $\left(^{1}\right)$
}

\author{
FERNANDA SALLES CUNHA PERES $\left({ }^{2}\right)$; ODAIR APARECIDO FERNANDES $\left({ }^{3}\right)$; \\ LUÍS CLÁUDIO PATERNO SILVEIRA $\left({ }^{4}\right)$; CHERRE SADE BEZERRA DA SILVA $\left(^{2}\right)$
}

\begin{abstract}
RESUMO
Foi avaliada a atratividade de Tagetes patula L. (cravo-de-defunto) sobre tripes (Thysanoptera) em cultivo protegido de melão. Foram estudadas a abundância, dominância, frequência e constância de espécies de tripes, utilizando-se análise faunística, análise de agrupamento (AA) e análise de componentes principais $(\mathrm{ACP})$. Nas extremidades das estufas com cultivo de melão foram plantadas faixas de cravo-de-defunto. Os insetos foram amostrados batendo-se três ponteiros das plantas de melão e todas as plantas inteiras de cravo-de-defunto $\left(1 \mathrm{~m}^{2}\right)$ em bandejas brancas. Os pontos de coleta foram: T. patula, melão consorciado com cravo-de-defunto e melão nas distâncias de $6 \mathrm{~m}, 12 \mathrm{~m}, 18 \mathrm{~m}$ e $24 \mathrm{~m}$ do cravo-de-defunto. Foram encontradas 11 espécies de tripes nas plantas de melão e cravo-de-defunto: Neohydatothrips sp., Frankliniella sp. 1, Frankliniella sp. 2, Frankliniella schultzei (Trybom), Scirtothrips sp., Caliothrips sp., Microcephalothrips sp., Franklinothrips vespiformis (Crawford), Arorathrips sp., Ceratothripoides sp. e Haplothrips sp. As espécies Neohydatothrips sp., Frankliniella sp. 1, F. schultzei e Caliothrips sp. foram dominantes. Estas foram utilizadas para determinar a atratividade do cravo-de-defunto. As análises demonstraram a existência de três grupos diferenciados: (1) cravo-de-defunto; (2) melão consorciado com T. patula e (3) melão nas distâncias de 6 m, $12 \mathrm{~m}, 18 \mathrm{~m}$ e $24 \mathrm{~m}$ do cravo-de-defunto. Por meio das AA e ACP foi possível verificar diferenças na abundância entre os pontos de coleta, mostrando a atratividade de T. patula sobre os tripes. Concluiu-se que o cravo-de-defunto pode ser utilizado como planta atrativa na cultura de melão para várias espécies de tripes.
\end{abstract}

Palavras-chave: Thysanoptera, planta armadilha, abundância, diversidade.

\section{ABSTRACT \\ MARIGOLD AS ATTRACTIVE PLANT FOR THRIPS IN PROTECTED ORGANIC MELON CULTIVATION}

The attractiveness of Tagetes patula L. (marigold) to thrips (Thysanoptera) in greenhoused melon was evaluated. Abundance, dominance, frequency, and constancy of thrips species were studied by using faunistic analysis, cluster analysis (CA) and principal component analysis (PCA). Marigold was planted only at the edge of the greenhouses. Insect sampling on melon plants was taken by shaking three vine growing ends on a white tray whereas similar procedure was used for sampling marigold but shaking all plants of $1 \mathrm{~m}^{2}$. Samplings points where on T. patula edge, on melon planted along with marigold, and on melon planted at $6 \mathrm{~m}, 12 \mathrm{~m}, 18 \mathrm{~m}$, and $24 \mathrm{~m}$ from marigold edge. Eleven thrips species were found on both melon and marigold plants: Neohydatothrips sp., Frankliniella sp. 1, Frankliniella sp. 2, Frankliniella

$\left.{ }^{1}\right)$ Parte da Dissertação de Mestrado da primeira autora. Recebido para publicação em 26 de junho de 2007 e aceito em 24 de abril de 2009.

$\left(^{2}\right)$ Mestre em Agronomia (Entomologia Agrícola), Universidade Estadual Paulista Júlio de Mesquita Filho -UNESP, Campus de Jaboticabal, Departamento de Fitossanidade, Rod. Prof. Paulo Donato Castellane, s/n, 14884-900 Jaboticabal (SP). E-mail: fernandasallesc@gmail.com; entomologista@gmail.com

${ }^{3}$ ) Departamento de Fitossanidade, UNESP/FCAV. E-mail: oafernandes@fcav.unesp.br $\left(^{*}\right)$ Autor correspondente. Bolsista CNPq.

$\left({ }^{4}\right)$ Departamento de Entomologia, Universidade Federal de Lavras, Caixa Postal 3037, 37200-000 Lavras (MG). E-mail: lcpsilveira@ufla.br 
schultzei (Trybom), Scirtothrips sp., Caliothrips sp., Microcephalothrips sp., Franklinothrips vespiformis (Crawford), Arorathrips sp., Ceratothripoides sp., and Haplothrips sp. The dominant species were: Neohydatothrips sp., Frankliniella sp. 1, F. schultzei, and Caliothrips sp. Analyses of the dominant species indicate three different groups according to abundance: (1) marigold, (2) melon along with T. patula, and (3) melon at 6m, 12m, $18 \mathrm{~m}$, and $24 \mathrm{~m}$ from marigold. Significant differences among sampling sites were verified by using CA and PCA. Therefore, T. patula is attractive to thrips and may be used as trap crop in melon cultivation for several thrips species.

Key words: Thysanoptera, trap crop, abundance, diversity.

\section{INTRODUÇÃO}

No Brasil estima-se que a produção sob o sistema de cultivo protegido seja atualmente de 17.000 hectares (Bueno, 2008). No Estado de São Paulo, aproximadamente $60 \%$ da área sob cultivo protegido corresponde ao plantio de hortaliças, enquanto o restante é destinado às flores de corte e envasadas (Tivelli, 1998). Dentre as principais hortaliças cultivadas estão o tomate, o pepino, a alface e o pimentão. Além disso, mais de 400 espécies de plantas ornamentais, principalmente rosas, crisântemos, gérberas, violetas, azaléias e gladíolos, são também cultivadas em sistema protegido (BUENO, 1999; IBRAFLOR, 2000).

O cultivo protegido proporciona produção o ano inteiro. Por outro lado, favorece o estabelecimento de pragas específicas, pois o ambiente tem alimento abundante e clima propício para propagação, aumentando as populações rapidamente (PARRELla et al., 1999). Por esse, há necessidade do aumento do número de aplicações de inseticidas, tornando o ambiente insalubre para os trabalhadores, e contribuindo para o desenvolvimento de resistência das pragas (OliveIra, 1995).

Um método de sucesso para o controle de pragas em cultivos protegidos é o manejo integrado (MIP), que utiliza vários métodos de controle, como o biológico, cultural e químico, além do manejo do agroecossistema, com uso de plantas armadilhas e plantas atrativas para inimigos naturais (PICANÇO e Guedes, 1999; Pallini et al., 2004). O uso de plantas de bordadura permite o aumento da diversidade vegetal, que é importante para a estabilidade da dinâmica populacional dos insetos fitófagos e favorece positivamente a biologia e dinâmica dos insetos benéficos, pela maior quantidade de alimento disponível para adultos (pólen e néctar), presença de presas alternativas e variedade de micro-habitat (Andow, 1991; Altieri e Nicholls, 1999; Altieri et al., 2003).

A entomofauna benéfica associada a plantas cultivadas tem sido avaliada para várias espécies, tais como milho, milheto, crotalária e nabo forrageiro (Silveira Neto, 2005). Assim, pode-se incrementar o controle biológico natural por meio do aumento de diversidade em bordaduras de cultivos. Estudos nessa linha de pesquisa são escassos, comparados aos estudos envolvendo liberação de inimigos naturais (controle biológico aplicado).

O consórcio de plantas é uma alternativa viável para os agricultores, pois permite economia de espaço na área cultivada. O consórcio de cravo-dedefunto (Tagetes erecta L. e Tagetes patula L.) com tomate teve sucesso. Estudos demonstraram que o consórcio com esta planta ornamental reduz os índices de afídeos, nematóides, moscas-brancas e plantas contaminadas com vírus, bem como pode causar o aumento da produção do tomateiro (MARTOWO e Rohama, 1987; Авid e Magbool, 1990; Zavaleta-Mejia e GOMES, 1995).

Portanto, pode-se utilizar plantas em bordadura para atuar como atrativas de inimigos naturais ou como armadilha para organismos fitófagos. Assim, o objetivo deste trabalho foi buscar estratégia para proteger o cultivo de melão orgânico da infestação de tripes por meio da avaliação da atração do cravo-de-defunto (T. patula) cultivado nas extremidades de estufas com melão.

\section{MATERIAL E MÉTODOS}

O experimento foi desenvolvido no Sítio Oyafuso, localizado no município de Araraquara (SP), certificado como produtor orgânico pelo Instituto Biodinâmico (IBD), em duas casas de vegetação tipo semiarco, com $60 \mathrm{~m}$ de comprimento e $7 \mathrm{~m}$ de largura, totalizando $420 \mathrm{~m}^{2}$ de cultivo protegido, cultivada com melão (Cucumis melo L., variedade Net Melon).

Os canteiros para cultivo foram compostos por: solo, cultura anterior incorporada (tomate e plantas invasoras), composto (torta de mamona, farelo de arroz, pó de rocha, cinza, calcário de ostra), água com melaço, ácido bórico, sulfato de zinco, molibdato de sódio e capim napier triturado. A casa de vegetação possuia quatro canteiros de $1 \mathrm{~m}$ de largura cada um. Um corredor de $1 \mathrm{~m}$ foi mantido entre os canteiros. 
Melão e cravo-de-defunto (T. patula) foram semeados em bandejas de isopor de 288 células contendo substrato Ecosolo ${ }^{\circledR}$ e, após 30 dias da emergência, as mudas foram transplantadas para a casa de vegetação. O espaçamento utilizado para o melão foi de $30 \times 30 \mathrm{~cm}$ e todos os canteiros da casa de vegetação foram transplantados. O cravo-dedefunto foi transplantado em duas faixas de $5 \mathrm{~m}$ nas extremidades das estufas, em espaçamento de $10 \times 30 \mathrm{~cm}$. No cruzamento destas faixas com os canteiros de melão, portanto, ocorreu a consorciação (Figura 1).

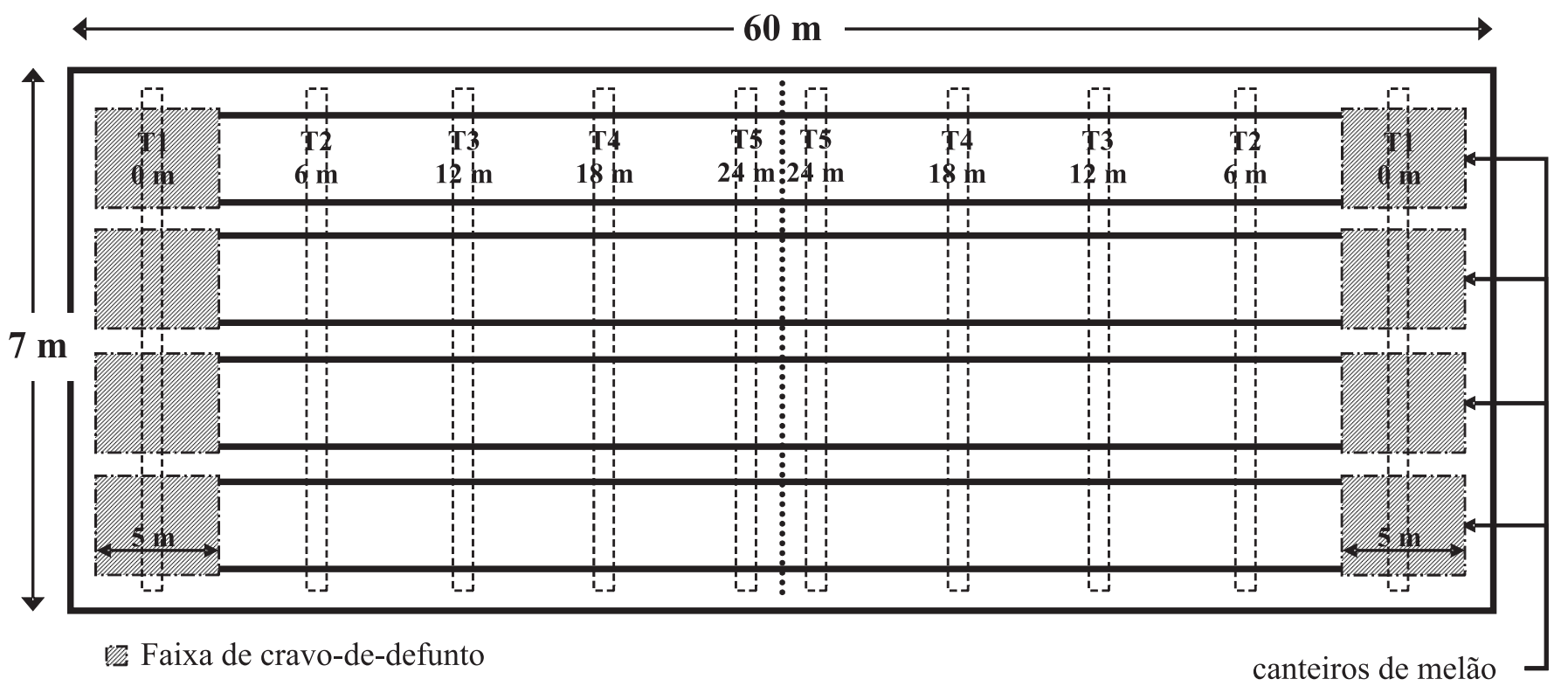

Figura 1. Croqui da área experimental indicando os pontos de coleta (Cravo-de-defunto; T1 [Melão consorciado com cravo-de-defunto]; T2, T3, T4 e T5 [Melão à distância de 6m, 12m, 18m e 24m do cravo-de-defunto]).

Os pontos de coleta foram a) cravo-de-defunto entre os canteiros com as plantas de melão; b) melão consorciado com cravo-de-defunto (T1); c) melão à distância de $6 \mathrm{~m}$ (T2); d) melão à distância de $12 \mathrm{~m}$ (T3); e) melão à distância de 18 m (T4) e f) melão à distância de $24 \mathrm{~m}$ do cravo-de-defunto (T5). As coletas de insetos foram feitas durante seis semanas. Cada amostra constitui-se de três ponteiros de plantas de melão e $1 \mathrm{~m}^{2}$ de plantas de cravo-de-defunto. As plantas foram batidas em bandeja branca e os insetos sugados em seguida, com sugador bucal. Após a sucção, os insetos foram armazenados em frascos contendo álcool $60 \%$ para posterior identificação. No laboratório foi feita a separação dos tripes, os quais foram encaminhados para identificação das espécies. As amostragens foram iniciadas 30 dias após o transplante do melão e realizadas até a frutificação (próximo à colheita), totalizando 144 amostras. Essas amostragens foram realizadas em janeiro e fevereiro de 2006.

Os dados foram analisados através dos índices faunísticos, por meio do programa Anafau (Silveira Neto et al., 2005), adotando-se o Método de Sakagami e Larroca para definir as classes de abundância, dominância, frequência e constância, com base em Silveira Neto et al. (1976). Além disso, as espécies dominantes dadas pela análise faunística foram submetidas à análise exploratória multivariada de dados realizando-se a Análise de Componentes Principais e Análise de Agrupamento, com o uso do programa Statistic 7.0 (STATSOFT, 2004). As análises de Agrupamentos (AA), hierárquica e não hierárquica (Kmédias) e de Componentes Principais (ACP) foram empregadas para avaliar a atratividade de T. patula nos diferentes pontos de coleta.

\section{RESULTADOS E DISCUSSÃO}

Foram constatadas onze espécies de tripes, sendo uma predadora, uma onívora e nove fitófagas. Arorathrips sp., Ceratothripoides sp. e Haplothrips sp. foram observadas exclusivamente em melão. Microcephalothrips sp., Frankliniella sp. 2 e Franklinothrips vespiformis (Crawford) foram coletadas somente em T. patula. As espécies Neohydatothrips sp., Frankliniella sp.1, Frankliniella schultzei (Trybom), Scirtothrips sp. e Caliothrips sp. estavam associadas às duas culturas (Tabela 1). Das onze espécies coletadas, quatro $(36,4 \%)$ foram acidentais $(<25 \%$ de presença nas coletas), uma $(9,1 \%)$ foi aleatória $(25 \%$ a $50 \%$ de presença nas coletas) e seis $(54,5 \%)$ foram constantes ( $>50 \%$ de presença nas coletas), de acordo com classificação proposta por Silveira Neto et al. (1976) (Tabela 2). 
Tabela 1. Espécies de tripes (Thysanoptera) encontradas em cultivo protegido de melão, em cujas extremidades foi realizado cultivo consorciado de cravo-de-defunto

\begin{tabular}{|c|c|c|c|}
\hline Espécies de Tripes & Categoria & Cravo-de-defunto & Melão \\
\hline Neohydatothrips sp. & Fitófago & $x$ & $x$ \\
\hline Haplothrips sp. & Fitófago & & $x$ \\
\hline Franklinothrips vespiformis & Predador & $X$ & \\
\hline Frankliniella sp.1 & Fitófago & $X$ & $X$ \\
\hline Frankliniella sp.2 & Fitófago & $X$ & \\
\hline Frankliniella schultzei & Onívoro & $X$ & $X$ \\
\hline Scirtothrips sp. & Fitófago & $X$ & $X$ \\
\hline Caliothrips sp. & Fitófago & $X$ & $X$ \\
\hline Arorathrips sp. & Fitófago & & $x$ \\
\hline Ceratothripoides sp. & Fitófago & & $X$ \\
\hline Microcephalothrips sp. & Fitófago & $X$ & \\
\hline
\end{tabular}

Destaca-se que a maioria das espécies registradas neste estudo $(81,82 \%)$ são fitófagas.

A abundância de espécies de tripes comuns foi maior em $T$. patula com $64,72 \%$, seguida do melão consorciado com $T$. patula $(12,44 \%)$ e de melão cultivado isoladamente a $6 \mathrm{~m}, 24 \mathrm{~m}, 12 \mathrm{~m}$ e $18 \mathrm{~m}$ de distância da bordadura de cravo-de-defunto com $8,70 \% ; 5,50 \% ; 4,32 \%$ e $4,32 \%$ respectivamente (Tabela 3). Destaca-se que Scirtothrips sp. foi constatado em cravo-de-defunto com $87,5 \%$ dos indivíduos e em melão consorciado com cravo-de-defunto com $12,5 \%$, não sendo observado no melão isoladamente. Esse fato pode mostrar a influência de $T$. patula sobre Scirtothrips sp. Embora, no Brasil, tripes não sejam pragas importantes no melão, Scirtothrips dorsalis
Hood é considerada uma praga prejudicial da cultura no Canadá, Flórida, Porto Rico e Caribe (Venette e Davis, 2004).

As espécies dominantes foram Neohydatothrips sp. $(27,32 \%$ dos indivíduos capturados) e Frankliniella sp. 1 (33,88\%), sendo muito abundantes, muito freqüentes e constantes, enquanto F. schultzei $(11,48 \%)$ e Caliothrips sp. $(12,02 \%)$ foram abundantes, freqüentes e constantes (Tabela 3). Vários gêneros de tripes registrados neste estudo têm sido observados em culturas importantes, como Neohydatothrips em feijão, soja e cravo-de-defunto; Caliothrips e Arorathrips em citros e monocotiledôneas; Microcephalothrips em dicotiledôneas e Scirtothrips em mandioca.

Tabela 2. Análise faunística das espécies de Thysanoptera observadas em cultivo de melão contendo as extremidades com cravo-de-defunto

\begin{tabular}{lccccc}
\hline Espécie & Indivíduos & Dominância & Abundância & Freqüência & Constância \\
\hline & $\%$ & & & & \\
Neohydatothrips & 27,32 & $\mathrm{D}$ & $\mathrm{MA}$ & $\mathrm{MF}$ & $\mathrm{W}$ \\
Haplothrips sp. & 6,56 & $\mathrm{ND}$ & $\mathrm{C}$ & $\mathrm{F}$ & $\mathrm{W}$ \\
Frankliniella vespiformis & 0,55 & $\mathrm{ND}$ & $\mathrm{R}$ & $\mathrm{PF}$ & $\mathrm{Z}$ \\
Frankliniella sp.1 & 33,88 & $\mathrm{D}$ & $\mathrm{MA}$ & $\mathrm{MF}$ & $\mathrm{W}$ \\
Frankliniella sp. & 1,64 & $\mathrm{ND}$ & $\mathrm{R}$ & $\mathrm{PF}$ & $\mathrm{Z}$ \\
Frankliniella schultzei & 11,48 & $\mathrm{D}$ & $\mathrm{C}$ & $\mathrm{F}$ & $\mathrm{W}$ \\
Scirtothrips sp. & 4,37 & $\mathrm{ND}$ & $\mathrm{C}$ & $\mathrm{F}$ & $\mathrm{W}$ \\
Caliothrips sp. & 12,02 & $\mathrm{D}$ & $\mathrm{C}$ & $\mathrm{F}$ & $\mathrm{W}$ \\
Arorathrips sp. & 1,09 & $\mathrm{ND}$ & $\mathrm{R}$ & $\mathrm{PF}$ & $\mathrm{Y}$ \\
Ceratothripoides sp. & 0,55 & $\mathrm{ND}$ & $\mathrm{R}$ & $\mathrm{PF}$ & $\mathrm{Z}$ \\
Microcephalothrips sp. & 0,55 & $\mathrm{ND}$ & $\mathrm{R}$ & $\mathrm{PF}$ & $\mathrm{Z}$ \\
\hline
\end{tabular}

$\mathrm{D}=$ dominante; $\mathrm{ND}=$ não dominante; $\mathrm{A}=$ abundante; $\mathrm{C}=$ comum; $\mathrm{R}=$ rara; $\mathrm{MA}=$ muito abundante; $\mathrm{F}=$ freqüente; $\mathrm{MF}=$ muito freqüente; $\mathrm{PF}=$ pouco freqüente; $\mathrm{W}=$ constante; $\mathrm{Y}=$ acessória; $\mathrm{Z}=$ acidental. 
Espécies de Haplothrips, por outro lado, podem causar distorção, enrolamento e galhas em folhas de eucalipto (Monteiro, 2002). F. schultzei é vetor de tospovirus e considerado praga de várias culturas, como algodoeiro, alface, melancia, tomateiro, pimentão e fumo (MonTeIro et al., 1999), embora possa utilizar ovos de outros ácaros para se alimentar (Milne e Walter, 1997) e, desse modo, atuar como predador. Esta espécie ocorreu no cultivo de melão e pode ser verificada também em girassol, roseira, amendoinzeiro, soja, batata, pepino, cebola, videira e abobrinha, mas aparentemente sem causar danos significativos, de acordo com Monteiro et al. (1999). Esse fato corrobora as observações de campo, pois não foi observado qualquer dano à cultura de melão na área de estudo.

Tabela 3. Proporção (\%) das espécies de Thysanoptera observadas em melão e T. patula cultivados em casa de vegetação, em diferentes condições

\begin{tabular}{|c|c|c|c|c|c|c|}
\hline Espécie & T. patula & $\begin{array}{c}\text { Melão consorciado } \\
\text { T.patula }\end{array}$ & $\begin{array}{l}\text { Melão a } 6 \mathrm{~m} \\
\text { de T. patula }\end{array}$ & $\begin{array}{c}\text { Melão a } 12 \mathrm{~m} \\
\text { de T. patula }\end{array}$ & $\begin{array}{c}\text { Melão a } 18 \mathrm{~m} \\
\text { de T.patula }\end{array}$ & $\begin{array}{r}\text { Melão a } 24 \mathrm{~m} \\
\text { de T. patula }\end{array}$ \\
\hline Neohydatothrips sp. & 92,68 & 4,88 & 2,44 & - & - & - \\
\hline Frankliniella sp. & 32,14 & 28,57 & 16,07 & 5,36 & 5,36 & 12,50 \\
\hline Frankliniella schultzei & 30,00 & 10,00 & 25,00 & 10,00 & 10,00 & 15,00 \\
\hline Scirtothrips sp. & 87,50 & 12,50 & - & - & - & - \\
\hline Caliothrips sp. & 81,25 & 6,25 & - & 6,25 & 6,25 & - \\
\hline Total & 64,72 & 12,44 & 8,70 & 4,32 & 4,32 & 5,50 \\
\hline
\end{tabular}

A maior abundância de espécies de tripes verificadas em cravo-de-defunto quando comparada ao melão revela que o uso dessa planta para aumento de diversidade vegetal pode ser interessante. Como não foram observados danos de tripes às plantas de melão, conclui-se que o uso de cravo nas bordaduras contribuiu para aumento da diversidade de fitófagos não-praga, que podem servir de alimento alternativo para diversos organismos entomófagos. Os tripes servem como presas para Coccinellidae (Coleoptera), Anthocoridae (Hemiptera) e Chrysopidae (Neuroptera), dentre outros insetos, além de hospedarem parasitóides Eulophidae (LATTIN, 2000; Tagashira e Hirose, 2001; DeligeOrgidis et al., 2005).

Dentre os hemípteros Anthocoridae, destacamse as espécies do gênero Orius Wolff. Esse grupo tem ampla distribuição mundial e é predador de pequenos artrópodes como tripes, ácaros, moscas-brancas e pulgões (Lattin, 2000). Em estudos com O. insidiosus, ELKASSABANY et al. (1996) verificaram que os insetos foram coletados em várias plantas invasoras com altas densidades de tripes. Isso mostra a importância da utilização dessas plantas como atrativo de tripes, formando um micro-habitat favorável a inimigos naturais, para que mantenham e migrem para a cultura, de modo que controlem outras pragas.

De acordo com os resultados verificados pela análise de agrupamento hierárquico (AA), ocorreram diferenças na densidade do tripes, entre os pontos de coleta, separando-se em três grupos distintos: I - T. patula, II - melão consorciado com T. patula e III - melão à distância de $6 \mathrm{~m}$ e a $24 \mathrm{~m}$
(Figura 2). No grupo III, pode-se observar no dendograma a separação em dois subgrupos: melão a $6 \mathrm{~m}$ e $24 \mathrm{~m}$ do cravo-de-defunto e o outro grupo melão a $12 \mathrm{~m}$ e a $18 \mathrm{~m}$ do cravo-dedefunto.

Pela análise K-médias, a maior densidade de tripes ocorreu no grupo I, seguido pelo grupo II e, em baixa densidade, pelo grupo III (Figura 3), confirmando a separação de grupos pela AA hierárquico. Isso mostra que $T$. patula abriga uma densidade alta de tripes, podendo funcionar como um reservatório de alimento alternativo para predadores e parasitóides.

Pelos resultados da análise dos componentes principais (ACP) para os seis pontos de coleta combinados, observou-se que os dois primeiros eixos explicaram $91 \%$ da variação total entre os caracteres; o primeiro componente explicou $79,75 \%$ da variação total e o segundo 11,25\% (Figura 4). O gráfico da projeção dos pontos de coleta no espaço dos dois primeiros componentes revela a existência de três grupos: um formado por cravo-de-defunto (I), outro por melão consorciado com $T$. patula e um terceiro, por melão a $6 \mathrm{~m}, 12 \mathrm{~m}, 18 \mathrm{~m}$ e $24 \mathrm{~m}$ de distância do cravode-defunto (Figura 4). Assim, através da ACP, confirmou-se a separação em três grupos. Percebe-se ainda que não houve diferença na diversidade e abundância de tripes nas plantas de melão cultivadas a diferentes distâncias do cravo-de-defunto. Portanto, não houve gradiente populacional das espécies ao longo da casa de vegetação. 


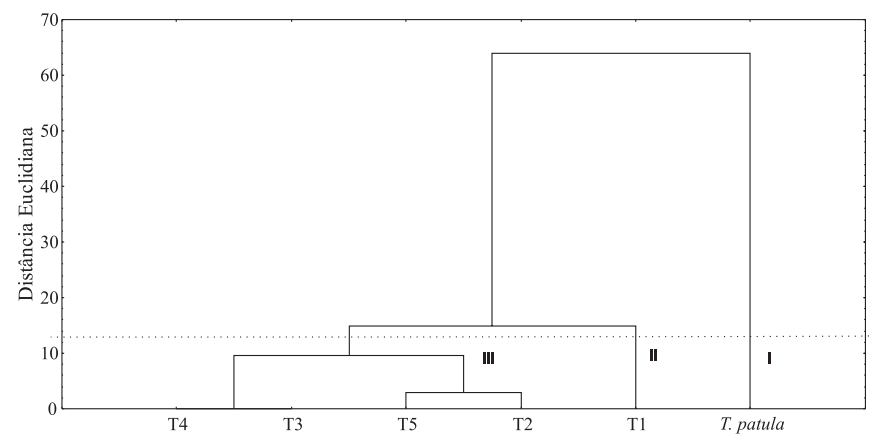

Figura 2. Análise de agrupamento hierárquico, considerando-se todos os pontos de coleta e espécies dominantes de tripes (Thysanoptera) amostrados. Os números I, II e III referem-se aos grupos formados. Grupo I: T. patula, II: Melão consorciado com T. patula (T1) e III: melão a 6 m (T2), 12 m (T3), 18 m (T4) e 24 m de T. patula (T5).

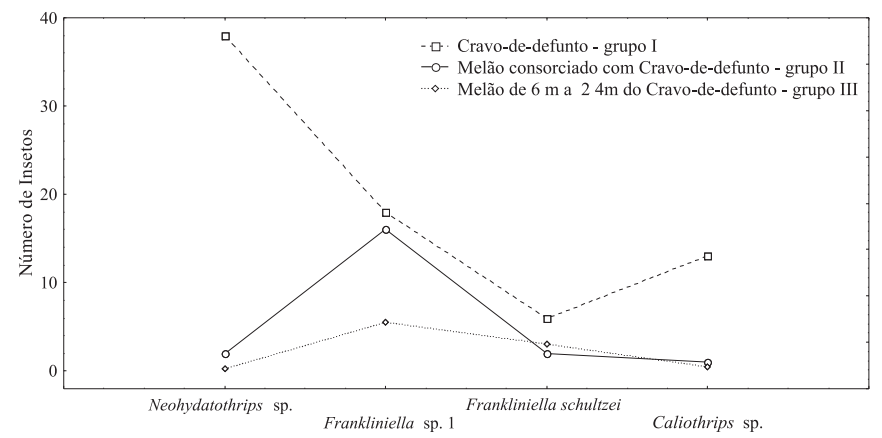

Figura 3. Análise de agrupamento não-hierárquico (Kmédias), considerando-se todos os pontos de coleta e espécies dominantes de tripes (Thysanoptera) amostrados.

O gráfico da projeção das variáveis no espaço dos dois primeiros componentes confirma a atratividade de T. patula sobre as espécies de tripes (Figura 4). O cravo-de-defunto foi o que mais contribuiu para o direcionamento das espécies no primeiro componente enquanto no segundo foi o consórcio de melão com o cravo-de-defunto (Tabela 4). Os padrões de ordenação entre as variáveis sugerem que o direcionamento está relacionado com o cravode-defunto. Portanto, isso pode ter sido causado pela sua atratividade aos insetos fitófagos.

Os três métodos multivariados permitiram verificar que existe atração dos tripes por T. patula. As espécies atraídas, no entanto, não provocaram danos à cultura do melão durante o período amostrado, que compreendeu tanto parte do período vegetativo, como do período reprodutivo da planta. Assim, T. patula pode ser recomendado como alternativa vegetal para o plantio em bordadura de cultivo protegido de melão visando atrair diversas espécies de tripes. Estudos futuros devem ser feitos para determinar a atratividade do cravo-de-defunto sobre outras espécies fitófagas e, conseqüentemente, sobre os entomófagos, pois, além de cultura-armadilha para tripes, o cravo-de-defunto pode ser também atrativo para inimigos naturais, que utilizariam estes recursos alimentares para se manterem na área de cultivo de melão.

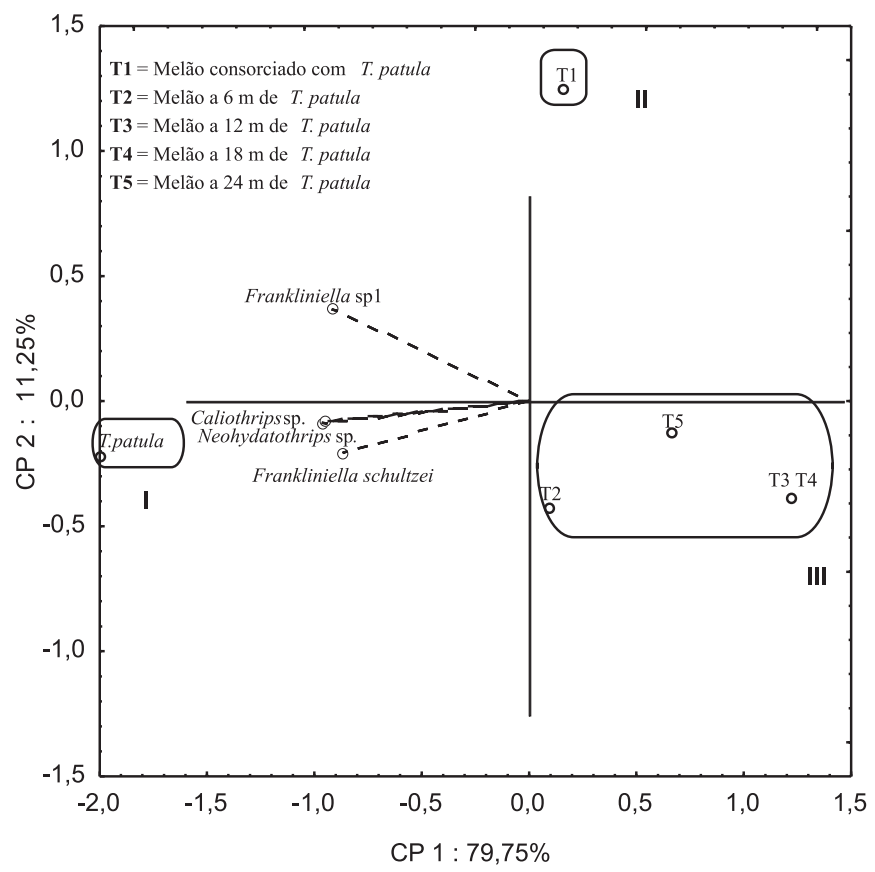

Figura 4. Componentes principais considerando-se todos os pontos e variáveis (espécies dominantes de tripes) amostrados. Os números I, II e III referem-se aos agrupamentos baseados no dendrograma. Grupo I ( $T$. patula), II (Melão consorciado com T. patula) e III (melão a $6 \mathrm{~m}, 12 \mathrm{~m}, 18 \mathrm{~m}$ e $24 \mathrm{~m}$ de $T$. patula) mostram a influência sobre os tripes.

Tabela 4. Fatores embasados na correlação da análise de componentes principais para as quatro espécies de tripes avaliadas

\begin{tabular}{lcc}
\hline Pontos de coleta & CP 1 & CP 2 \\
\hline Cravo-de-defunto & $-1,96500$ & $-0,263435$ \\
Melão consorciado & 0,13015 & 2,007754 \\
com cravo-de-defunto & & \\
Melão a 6 m do cravo-de-defunto & 0,03554 & $-0,591391$ \\
Melão a 12 m do cravo-de-defunto & 0,68282 & $-0,517663$ \\
Melão a 18 m do cravo-de-defunto & 0,68282 & $-0,517663$ \\
Melão a 24 m do cravo-de-defunto & 0,43368 & $-0,117602$ \\
\hline
\end{tabular}




\section{CONCLUSÃO}

O cravo-de-defunto, T. patula, serve como planta atrativa para Neohydatothrips sp.; Frankliniella sp. 1; Caliothrips sp. e F. schultzei (Thysanoptera) e, dessa forma, pode ser recomendado para plantio na bordadura do cultivo principal para reduzir a infestação por espécies fitófagas de tripes.

\section{AGRADECIMENTOS}

Os autores agradecem ao Sr. Marcelo Oyafuso, produtor orgânico de Araraquara (SP), pela colaboração e cessão da área para estudo e à Coordenação de Aperfeiçoamento de Pessoal de Nível Superior (CAPES) pelo financiamento da bolsa de estudos do primeiro autor. Identificação de tripes com recursos da Fapemig. Agradecem os dois revisores anônimos pelas contribuições.

\section{REFERÊNCIAS}

ABID, M.; MAGBOOL, M.A. Effects of inter-cropping of Tagetes erecta on root-knot disease and growth of tomato. International Nematology Network Newsletter, v.7, p. 41-42, 1990.

ALTIERI, M.A.; NICHOLLS, C.I. Biodiversity, ecosystem function, and insect pest management in agricultural systems. In: COLLINS W.W.; QUALSET C.O. (Ed.). Biodiversity in agroecosystems. Boca Raton: CRC Press, 1999. p. 69-84.

ALTIERI, M.A.; SILVA, E.N.; NICHOLLS, C.I. O papel da biodiversidade no manejo de pragas. Ribeirão Preto: Holos, 2003. 226p.

ANDOW, D.A. Vegetational diversity and arthropod population response. Annual Review of Entomology, v.36, p.561-586, 1991.

BUENO, V.H.P. Protected cultivation and research on biological control of pests in greenhouses in Brazil. IOBC/ WPRS Bulletin, v. 22, p.21-24, 1999.

BUENO, V.H.P. Identificação e controle pragas sob sistema de cultivo protegido. Revista Plasticultura, v.1, n.3, p.34-35, 2008.

DELIGEORGIDIS, P. N.; IPSILANDIS, C.G., VAIOPOULOU, M.; KALTSOUDAS, G.; SIDIROPOULOS, G. Predatory effect of Coccinella septempunctata on Thrips tabaci and Trialeurodes vaporariorum. Journal of Applied Entomology, v.129, p.246249, 2005.

ELKASSABANY, E.; RUBERSON, J.R.; KRING T. Seasonal distribution and overwintering of Orius insidiosus (Say) in Arkansas. Journal of Entomological Science, v.31, p.7688, 1996.

IBRAFLOR. O desenvolvimento da floricultura brasileira. Relatório Floricultura Brasileira 19. 2000. Disponível em: $<\mathrm{http}$ : www.flortec.com.br/ Artigo04.htm.>. Acesso em: 8 jan. 2007.
LATTIN, J.D. Economic importance of minute pirate bugs (Anthocoridae). In: SCHOEFER, C.W.S.; PANIZZI A.R. (Ed.). Heteroptera of economic importance. New York: CRC Press, 2000. p.607-637.

MARTOWO, B.; ROHANA, D. The effect of intercropping of pepper (Capsicum annum L.) with some vegetable crops on pepper yield and disease incidence caused by Meloidogyne spp. Buletin Penelitian Hortikultura, v.15, p.55-59, 1987.

MILNE, M.; WALTER, G. H. The significance of prey in the diet of the phytophagous thrips, Frankliniella schultzei. Ecological Entomology, v.22, p.74-81, 1997.

MONTEIRO R.C. Estudos taxonômicos de tripes (Thysanoptera) constatados no Brasil, com ênfase no gênero Frankliniella. 1999. 144f. Tese (Doutorado em Agronomia) Escola Superior de Agricultura "Luiz de Queiroz", Universidade de São Paulo, Piracicaba, 1999.

MONTEIRO, R.C. The Thysanoptera fauna of Brazil. In: INTERNATIONAL SYMPOSIUM ON THYSANOPTERA, 7., Reggio Calabria. Proceedings of the thrips and tospoviruses, Reggio Calabria, 2002. p.325-340.

OLIVEIRA, M.R.V. O emprego de casas de vegetação no Brasil: vantagens e desvantagens. Pesquisa Agropecuária Brasileira, v.30, p.1049-1060, 1995.

PALINNI, A.; VENZON, M.; OLIVEIRA H.G.; FADINI M.A.M. Manejo Integrado de Pragas em cultivo protegido. In: AGUIAR, R.L.;DAREZZO, R.J.;ROZANE, D.E.;AGUILERA, G.A.H.;SILVA, D.J.H. (Ed.). Cultivo em ambiente protegido: histórico, tecnologia e perspectivas. Viçosa: UFV, 2004. p.207-220.

PARRELLA, M.P.; HANSEN, L.S.; VAN LENTEREN, J.C. Glasshouse environments. In: BELLOWS, T. S.; FISHER, T.W.; CALTAGIRONE, L.E.; DAHLSTEN, D.L.; HUFFAKER, C.; GARDH, G. Handbook of biological control: principles and applications of biological control. New York: Academic Press, 1999. p.819-839.

PICANÇO, M.; GUEDES, R.N.C. Manejo Integrado de Pragas no Brasil: situação atual, problemas e perspectivas. Ação Ambiental, v.2, p.23-27, 1999.

SILVEIRA NETO, S.; NAKANO, O.; BARBIN, D.; VILLA NOVA, N.A. Manual de ecologia dos insetos. Piracicaba: Ceres, 1976. 419 p.

SILVEIRA NETO, S.; HADDAD, M.L.; MORAES, R.C.B.; LAI REYES, A.E. ANAFAU - análise faunística. Piracicaba: ESALQ, 2005. Disponível em: <http:www.esalq.usp.br>. Acesso em: 10 jan. 2007.

STATSOFT, Inc. Statistica: data analysis software system, version 7., 2004.

TAGASHIRA, E.; HIROSE Y. Development and reproduction of Ceranisus menes (Hymenoptera: Eulophidae), a larval parasitoid of thrips: effects of two host species, Frankliniella intonsa and Thrips palmi (Thysanoptera: Thripidae). Applied Entomology and Zoology, v.36, p.237-241, 2001. 
TIVELLI, S.W. Manejo do ambiente em cultivo protegido. In: GOTO, R.; TIVELLI, S.W. Produção de hortaliças em cultivo protegido: condições subtropicais. São Paulo: Fundação Editora Unesp, 1998. p.15-30.

VENETTE, R.C.; DAVIS E.E. Chilli thrips/yellow thrips, Scirtothrips dorsalis Hood (Thysanoptera: Thripidae) mini pest risk assessment. St. Paul: University of Minnesota, 2004. 31 p.

ZAVALETA-MEJÍA, E.; GOMEZ, R. O. Effect of Tagetes erecta L.-tomato (Lycopersicon esculentum Mill.) intercropping on some tomato pests. Fitopatologia, v.30, p.35-46, 1995. 